\title{
Complicações ginecológicas e condutas preventivas em pacientes pós- transplante de células-tronco hematopoiéticas
}

\author{
Gynecological complications and preventive conduct in patients post-transplantation \\ hematopoietic stem cells
}

\section{Complicaciones ginecológicas y conducta preventiva en pacientes post-trasplante de células madre hematopoyéticas}

Janaina Cerqueira de Paiva ${ }^{1 *}$, Amanda Ferreira Silva², Ana Laura Campos Valadares ${ }^{3}$, Beatriz Xavier Machado ${ }^{4}$, Izabella de Castro Cabral Kneip ${ }^{5}$, Karina Santos de Faria ${ }^{6}$, Leonardo Fávaro Pereira $^{7}$, Marcela Chiriano ${ }^{8}$, Mariana Calheiros Poloni ${ }^{9}$, Thales Miranda Sales ${ }^{10}$.

\section{RESUMO}

Objetivo: Descrever e discutir o desenvolvimento de complicações ginecológicas após o Transplante de Células-Tronco Hematopoiéticas (TCTH), entre elas a Doença do Enxerto Contra o Hospedeiro (DECH). Revisão Bibliográfica: Esta complicação ocorre quando há um ataque imunológico das células da medula transplantada contra o receptor e afeta órgãos como pulmão, pele, trato gastrointestinal e mucosas. Além disso, a DECH representa uma das principais causas de morbimortalidade ocasionada pelo TCTH e isso ocorre devido à falta de acompanhamento médico regular e de informações sobre as condutas preventivas pré e pós transplante. Quando ocorrem manifestações ginecológicas, os tratamentos são baseados em terapia hormonal sistêmica, fármacos tópicos e dilatadores vaginais. Considerações finais: A partir desta revisão narrativa sobre o tema, reforça-se a importância da regularidade dos exames ginecológicos em pacientes que se submeteram a TCTH e, considerando que os achados são majoritariamente clínicos e nem sempre evidenciados em biópsia, cabe aos ginecologistas se atentarem à conduta para com essas mulheres, orientando-as acerca de possíveis complicações, visto que muitas vezes são relevadas ante o desconhecimento ou insegurança das pacientes.

Palavras-chave: Transplante de medula óssea, Complicações, Doença do enxerto contra hospedeiro.

\section{ABSTRACT}

Objective: Describe and discuss the development of gynecological complications after Hematopoietic Stem Cell Transplantation (HSCT), including Graft Versus Host Disease (GVHD). Literature review: This complication occurs when there is an immune attack by the cells of the transplanted spinal cord against the recipient and affects organs such as the lung, skin, gastrointestinal tract and mucous membranes. In addition, GVHD represents one of the main causes of morbidity and mortality caused by HSCT andthis is due to the lack of regular medical follow-up and information on preventive measures before and after transplant. When gynecological manifestations occur, treatments are based on systemic hormonal therapy, topical drugs and

\footnotetext{
${ }^{1}$ Centro Universitário UNIFAMINAS (UNIFAMINAS), Muriaé - MG. *E-mail: janacpaiva@gmail.com 2Universidad Autónoma San Sebastián (UASS), San Lorenzo - PY.

${ }^{3}$ Centro Universitário Presidente Antônio Carlos (UNIPAC), Juiz de Fora - MG.

${ }^{4}$ Centro Universitário de Volta Redonda (UniFOA), Volta Redonda - RJ.

${ }^{5}$ Centro Universitário de Caratinga (UNEC), Caratinga - MG.

${ }^{6}$ Universidade de Vassouras (UV), Vassouras - RJ.

7 Universidade Federal do Espírito Santo (Ufes), Vitória - ES.

8 Universidade do Oeste Paulista (Unoeste), Jaú - SP.

'Universidade Estadual do Oeste do Paraná (Unioeste), Cascavel - PR.

10Universidade Federal de Ouro Preto (Ufop), Ouro Preto - MG.
} 
vaginal dilators. Final considerations: From this narrative review on the theme, the importance of regular gynecological exams in patients who underwent HSCT is reinforced and, considering that the findings are mostly clinical and not always evidenced in biopsy, it is up to the gynecologists to pay attention to the conduct for with these women, guiding them about possible complications, since they are often revealed in the face of patients' lack of knowledge or insecurity.

Key words: Bone marrow transplant, Complications, Graft versus host disease.

\section{RESUMEN}

Objetivo: Describir y discutir el desarrollo de complicaciones ginecológicas después del Trasplante de Células Madre Hematopoyéticas (TCMH), incluida la Enfermedad de Injerto Contra Huésped (EICH). Revisión bibliográfica: Esta complicación se produce cuando hay un ataque inmunológico de las células de la médula espinal trasplantada contra el receptor y afecta a órganos como el pulmón, la piel, el tracto gastrointestinal y las mucosas. Además, la EICH representa una de las principales causas de morbimortalidad por TCMH y esto se debe a la falta de seguimiento médico periódico y de información sobre las medidas preventivas antes y después del trasplante. Cuando se producen manifestaciones ginecológicas, los tratamientos se basan en hormonoterapia sistémica, fármacos tópicos y dilatadores vaginales. Consideraciones finales: A partir de esta revisión narrativa sobre el tema, se refuerza la importancia de los exámenes ginecológicos periódicos en las pacientes sometidas a TCMH y, considerando que los hallazgos son mayoritariamente clínicos y no siempre evidenciados en biopsia, corresponde a los ginecólogos prestar atención a la conducta de con estas mujeres, orientándose sobre posibles complicaciones, ya que muchas veces se revelan ante el desconocimiento o la inseguridad de las pacientes.

Palabras clave: Trasplante de médula ósea, Complicaciones, Enfermedad de Injerto contra huésped.

\section{INTRODUÇÃO}

O Transplante de Células-Tronco Hematopoiéticas (TCTH) consiste na substituição de uma medula óssea doente ou deficitária por células progenitoras hematopoéticas com o objetivo de reconstituição da sua função (INCA, 2018). O TCTH pode ser do tipo alogênico, indicado para o tratamento de leucemias agudas e falências medulares ou do tipo autólogo, para afecções como linfomas. Porém, após o transplante de células-tronco hematopoéticas alogênicas (TCTH-alo) a Doença do Enxerto Contra o Hospedeiro (DECH) é uma possível complicação em que as células imunes funcionais da medula óssea transplantada atacam as células do receptor (MACHADO AN, 2019).

A DECH pode se manifestar de forma aguda ou crônica em pacientes que receberam transplantes alogênicos. Inicialmente, sua classificação era baseada apenas no tempo de manifestação da doença após o transplante. No entanto, no mais recente consenso do National Institutes of Health (NIH), foi definido que a determinação clínica de $\mathrm{DECH}$ em aguda ou crônica deveria considerar os tipos de manifestações apresentadas após o TCTH. Os principais alvos da DECH são pele, pulmão, fígado, trato gastrointestinal, mucosas e olhos. No entanto, existem também manifestações ginecológicas, que são menos prevalentes (JAGASIA MH, et al., 2015; CIAVATTINI A e CLEMENTE N, 2015; SOARES AC, 2016).

A maioria dos sintomas ginecológicos pós-transplante de medula óssea surgem em decorrência da DECH. As alterações escleróticas vulvovaginais podem culminar na estenose introital e no fechamento vaginal completo, o que gera dispareunia e dificulta as relações sexuais. A Insuficiência Ovariana Primária (POF) é outra complicação da DEHC que impacta significativamente na vida da mulher, causando infertilidade, fogachos, instabilidade de humor e risco de osteoporose. Ademais, deve-se atentar ao alto risco de infecções relacionadas ao Papilomavírus Humano (HPV) (MURPHY J, et al., 2019; JACOBSON M, et al., 2019).

As queixas mais comuns das mulheres acometidas por essa problemática são ressecamento vaginal, prurido, queimação, disúria e dispareunia. Há, ainda, mulheres que relatam perda de libido, menor prazer nas 
relações sexuais e irritação vaginal. Dessa forma, tais complicações resultam em perda da qualidade de vida, bem como da capacidade sexual, sendo o acompanhamento ginecológico fundamental para detectá-las precocemente e tratá-las de maneira adequada (DYER G, et al., 2016; HIRSCH P, et al., 2019; MACHADO AN, et al., 2019).

O acompanhamento ginecológico pré, peri e pós-transplante torna-se imprescindível para que as pacientes transplantadas possam ser triadas para sintomas da DECH e avaliadas adequadamente, a fim de que elas tenham acesso às condutas preventivas das possíveis complicações e realizem um tratamento qualificado. Nas manifestações ginecológicas da $\mathrm{DECH}$, o tratamento é baseado em terapia de reposição hormonal sistêmica, dilatadores vaginais e fármacos adequados, visando diminuir a incidência de acometimento vaginal e proporcionar melhor qualidade de vida da mulher transplantada (CIAVATTINI A e CLEMENTE N, 2015). Diante disso, o presente estudo visa analisar as complicações ginecológicas resultantes da DECH em pacientes pós TCTH e avaliar a relevância das condutas preventivas e complementares para que os prognósticos sejam os mais positivos possíveis.

\section{REVISÃO BIBLIOGRÁFICA}

O TCTH se fundamenta como um método de tratamento para diversas patologias e que normalmente leva o paciente a procedimentos de quimioterapia e/ou radioterapia, seguido da infusão de células progenitoras ou células-tronco hematopoéticas. Estas podem ser obtidas através de doações provenientes das células progenitoras do próprio paciente (autólogo), ou de um doador previamente selecionado por testes de compatibilidade (alogênico) ou de células progenitoras provenientes de um irmão univitelino (singênico), podendo ser obtidas por meio de medula óssea, sangue periférico e sangue umbilical (JACOBSON M, et al., 2019; FIGUEIREDO TB, et al., 2018).

Uma das principais complicações do Transplante de Células-Tronco Hematopoéticas alogênicas (TCTHalo) é a chamada Doença do Enxerto Contra o Hospedeiro crônica (DECHc), definida como um distúrbio imunológico sistêmico resultante do enxerto de células imunocompetentes de um doador para um hospedeiro imunocomprometido. Ela ocorre quando há o ataque imunológico das células da medula óssea transplantada contra o receptor, tendo como alvos principais pele, pulmão, fígado, trato gastrointestinal, membranas, mucosas ou olhos, podendo, inclusive, acometer simultaneamente tais sistemas. Esta complicação tardia da haste hematopoiética alogênica normalmente ocorre meses após o procedimento de transplante com um início insidioso (HIRSCH P, et al., 2012; STRATTON P, et al., 2012; SOARES AC, et al., 2016).

A patogênese da $\mathrm{DECHc}$ envolve uma resposta imune complexa, mediada por células $T$, na qual as células enxertadas reconhecem o hospedeiro como estranho, podendo causar, ginecologicamente, alterações que envolvam vagina e vulva. $O$ hipoestrogenismo resultante da insuficiência ovariana prematura induzida por quimioterapia também pode causar dor vulvar ou vaginal e irritação após o TCTH. Ainda que estes sintomas possam interferir na qualidade de vida da paciente, principalmente na intimidade sexual, raramente mulheres relatam tais fatos aos profissionais de saúde e, portanto, a DECHc acaba se tornando subdiagnosticada (STRATTON P, et al., 2012).

Corson SL, et al. (1982) fizeram o primeiro relato de DECH vulvovaginal e mais recentemente, outros estudos descreveram a ocorrência de doença DECH vulvar após transplante tradicional de TCTH. Apesar de o primeiro caso ter sido diagnosticado em 1982, acredita-se que a DECH genital seja subnotificada e subinvestigada o que corrobora a carência de dados de alta qualidade, resultando em uma variada metodologia de diagnóstico (JACOBSON M, et al., 2019).

Ainda de acordo com Jacobson M, et al. (2019) a DECH genital é comumente sincrônica (73-100\%) com DECHc sistêmica, podendo apresentar-se isoladamente também. Dentre os fatores de risco incluem-se: relação doador/receptor; uso de corticosteroides sistêmicos; parâmetro de hematócrito sanguíneo, história prévia de DECH aguda, coexistência de alterações em outras mucosas e aumento da idade. Refere-se tempo médio para a apresentação dos primeiros sintomas entre sete meses e dois anos, tendo relato de diagnóstico até sete anos pós-TCTH. 


\section{Complicações diagnósticas}

Uma das dificuldades encontradas no estudo das disfunções gonadais pela DECH é a variação da população desde o recrutamento inicial, no exame ginecológico pré-transplante, até a completude do tempo estimado para o fim do acompanhamento, que geralmente abrange o período de um ano ou mais. Isso acontece pois, algumas pacientes, cursam com recidivas da doença primária ou morte precoce (SMITH KNUTSSON E, et al., 2018). Outro fator importante é o subdiagnóstico, comum nos casos de pacientes assintomáticas, com ou sem sinais da doença, que podem achar irrelevantes certos tipos de informação ou até se sentirem inseguras, se negando a relatar sintomas ginecológicos. Todos esses fatores, associam-se ao fato de que muitos profissionais se encontram despreparados em revelar a verdadeira importância da busca desses sintomas específicos (NOERSKOV KH, et al., 2016).

Segundo Smith KE, et al. (2018) e Lev-Sagie A, et al. (2020), o ideal é que a paciente passe por consultas regulares, tanto na etapa pré-transplante quanto após o TCTH, independentemente de haverem sintomas, pois sabe-se que os sinais podem estar presentes e passar despercebidos, especialmente nas pacientes que não sejam sexualmente ativas. Os autores também propõem que toda paciente submetida a esse transplante deve realizar acompanhamento de três a quatro vezes durante o primeiro ano, a cada seis a doze meses nos dois anos seguintes, e anualmente a partir de três anos do procedimento, de forma contínua ao longo da vida, garantindo a detecção de acometimentos secundários, inclusive malignidades.

Além disso, os estudos sugerem que um especialista em DECH deve ser consultado ao aparecimento de quaisquer sinais e sintomas indicativos da doença, o que é reforçado pela pesquisa de Noerskov $\mathrm{KH}$, et al. (2016) que, ao estudarem a relação entre a sexualidade e a função sexual pós-TCTH em homens e mulheres, apontaram que apesar dos homens terem maior redução de função sexual ao longo do tempo, a recuperação posterior é mais rápida e possível, enquanto as manifestações femininas cursam com estabilidade da disfunção sexual desde o primeiro momento após o procedimento, com menor probabilidade de recuperação da função. Dessa maneira, denota-se a necessidade de esclarecer, desmistificar e aconselhar essas pacientes ainda no ambiente pré-operatório, com acompanhamento dos sintomas genitais e a possibilidade de intervenção precoce, garantindo melhor qualidade de vida (LEV-SAGIE A, et al., 2020).

Inicialmente, a classificação da DECH era apenas temporal, e considerava manifestação aguda quando essa acontecia em menos de 100 dias, e crônica quando ocorria em tempo superior a esse período. Porém, não havia discriminação entre os tipos de sinais e sintomas que ocorriam em cada período. No entanto, no mais recente consenso do NIH (2015), foi definido que a determinação clínica de DECHa (aguda) ou DECHc (crônica) deveria considerar os tipos de manifestações apresentadas após o TCTH. Isto posto, os órgãos e sistemas mais frequentemente acometidos pelo $\mathrm{DECH}$ foram listados e, para cada um, definidos os sinais $\mathrm{e}$ sintomas, subclassificados em comuns (que podem ocorrer em ambas as fases), diagnósticos (satisfatórios para o diagnóstico de $\mathrm{DECHc}$ ), distintos (estão presentes, mas quando isolados são insuficientes para determinar o diagnóstico de DECHc), além de manifestações raras, controversas e não específicas e/ou de DECHc que não podem ser utilizadas para firmar o diagnóstico de DECHc (JAGASIA MH, et al., 2015).

Embora não haja um período exato para detecção dos primeiros sinais e sintomas genitais, a partir da realização do TCTH as manifestações clínicas normalmente se iniciam em alguns meses, sendo o aparecimento após um ano do procedimento considerado tardio. As chances de desenvolver a doença são proporcionalmente maiores com o passar do tempo e não raramente se encontra associada a $\mathrm{DECH}$ em outros sistemas, como pele, olhos e boca. Estima-se, ainda, que a maioria das mulheres que se submetem a um TCTH desenvolverão a DECHc. Estudos a longo prazo consideram que a prevalência de DECHc genital em três anos será maior do que a $\mathrm{DECHc}$ extragenital e, embora geralmente se apresentem de forma concomitante, a segunda cursa com manifestações mais precoces do que a primeira (SMITH KE, et al, 2018).

Sutani A, et al. (2019) constataram o desenvolvimento de hipogonadismo hipergonadotrófico, com elevação dos níveis de gonadotrofina e redução das dosagens de hormônio anti-Mülleriano e estradiol, com consequente falência ovariana primária após o transplante, o que pode justificar a maior incidência da DECHc com manifestação genital. Também por esse motivo, é possível compreender a dificuldade na retomada da função sexual, que pode levar até seis anos decorridos do pós-transplante. Mesmo que os fatores fisiológicos 
e ambientais tenham grande influência, as queixas mais frequentes são relacionadas a alterações nos níveis hormonais, como redução da libido e dispareunia, que exigem terapêutica sistêmica e local (DYER G, et al., 2016).

Por conseguinte, é de extrema importância que o procedimento seja precedido e sucedido de assistência contínua da saúde genital, preservando a integridade do sistema reprodutor e a fertilidade de mulheres em todas as faixas etárias, uma vez que, quando realizado em jovens púberes, o transplante demanda terapia de reposição hormonal e, em mulheres mais velhas, o acompanhamento pode evitar o desenvolvimento de menopausa prematura (SUTANI A, et al., 2019; SMITH KE, et al., 2018).

\section{Condutas preventivas pré e pós transplante}

A DECH representa uma das principais causas de morbimortalidade decorrente do TCTH e pode resultar em graves complicações ginecológicas nas pacientes do sexo feminino. As manifestações genitais da DECH são complicações subnotificadas que afetam, pelo menos, $25 \%$ das pacientes que passam pelo TCTH, as quais apresentam diversos sintomas na vulva e vagina, causando malefícios que podem se tornar irreversíveis como insuficiência ovariana, disfunção sexual e infertilidade (MACHADO AN, et al., 2019). A fim de evitar tais complicações, as condutas preventivas pré e pós TCTH são indispensáveis.

Antes de realizar o TCTH, a paciente precisa passar pela fase de condicionamento, que consiste em preparar o corpo do receptor para a chegada das células tronco hematopoiéticas transplantadas. Nessa etapa, a paciente é submetida a sessões de quimioterapia e radioterapia que têm a finalidade de destruir as células imunes do hospedeiro, facilitando a admissão das células tronco recebidas por meio do transplante. Os medicamentos utilizados na quimio e radioterapia em altas doses, geram insuficiência ovariana e, consequentemente, diminuição da libido, dispareunia, infertilidade, disfunção sexual, secura e fibrose vaginal. Para evitar esse dano, deve-se fazer a administração precoce de terapia hormonal, de forma tópica ou sistêmica, com o intuito de conservar as características fisiológicas normais do trato genital e a arquitetura da mucosa vaginal (MACHADO AN, et al., 2019).

Sabe-se que muitas mulheres que passam pelo TCTH acabam sofrendo de falência ovariana prematura devido à fase de condicionamento, idade e complicações pós-transplante, e uma minoria delas consegue recuperar a função ovariana por um tempo limitado antes de entrar na menopausa. Sendo assim, é indicado o fracionamento da radiação para reduzir o risco em comparação com a radiação não fracionada (MAJHAIL NS, et al., 2012). Além disso, como grande parte das pacientes que passam pelo TCTH tornam-se inférteis ou possuem dificuldades relacionadas à concepção, para medida preventiva da fertilidade é indicado o armazenamento de tecido ovariano, de oócito ou de embrião (LAMBERTINI M, et al, 2016)

No entanto, grande parte da DECH com manifestações genitais leves, que envolvem a vulva ou vagina, podem ser assintomáticas e identificadas apenas por meio de um exame clínico. Sendo assim, mesmo as pacientes que não apresentam sintomas devem fazer exames ginecológicos de rotina de três a quatro vezes durante o primeiro ano e, depois, a cada seis ou doze meses após o transplante para prevenir o aparecimento de quadros mais graves (SMITH KE, et al., 2018).

Frequentemente, a DECH genital nas mulheres está associada a manifestações em outros órgãos, como a pele, superfícies da mucosa - boca, olhos e trato gastrointestinal - e pode aparecer mesmo que muito tempo tenha se passado após a manifestação inicial da DECH. Em cerca de $70 \%$ das pacientes, os sinais afetam apenas a vulva e, em $30 \%$, a vulva e a vagina, sendo que a manifestação vulvar geralmente precede a vaginal (KORNIK RI e RUSTAGI AS, 2017). Esse espaço temporal entre sintomas vulvares e vaginais permite o início de medidas profiláticas que impedem a ocorrência de casos mais graves, como dilatador vaginal revestido com estrogênio tópico ou clobetasol. Estudos demonstram, ainda, que o uso tópico do creme estriol $1 \mathrm{mg}$ ou supositórios de $1 \mathrm{mg}$ em dias alternados são indicados tanto para minimizar a atrofia epitelial vaginal resultante da falência ovariana e ação dos corticóides, quanto para manter a lubrificação e elasticidade da vagina, de forma a oferecer uma melhor saúde sexual à mulher (MACHADO AN, et al., 2019). Dessa forma, reforça-se a importância de que mulheres sintomáticas sejam encaminhadas para consultas com um ginecologista experiente. 
É importante frisar que é preciso estimular o retorno às atividades sexuais regulares ou o uso de dilatadores, anéis de liberação de estrogênio e a manutenção da atividade sexual regular para prevenir formação de sinéquias vaginais, estreitamento vaginal e estenose. Entretanto, é indicado evitar relações sexuais, dilatação ou lise cirúrgica das sinéquias quando a inflamação é aguda, uma vez que pode resultar no agravamento da inflamação. As sinéquias vaginais são facilmente separadas manualmente sob anestesia ou de forma cirúrgica e sua recorrência é comum e, por esse motivo, deve-se estimular as pacientes a fazerem autoexames digitais algumas vezes por semana. Da mesma forma, pacientes que recebem estrogênios locais na alta do departamento de transplante apresentam uma redução significativa na secura vaginal e dispareunia, além de minimização dos sintomas da menopausa que mimetizam a $D E C H$, fato que ajuda o diagnóstico em um estágio inicial ((MACHADO AN, et al., 2019; KLASA Ł, et al., 2020).

Além disso, o uso prolongado de imunossupressores para o tratamento da $\mathrm{DECH}$ genital é um fator de risco para a displasia associada ao HPV. Por isso, é recomendada a realização de exames ginecológicos regulares anuais, incluindo citologia cervical. No caso de lesões genitais suspeitas, faz-se a colposcopia e biópsia. Além disso, a vacinação contra HPV deve ser oferecida a mulheres jovens após o transplante para reduzir o risco de reativação viral e o teste de HPV deve ser repetido a cada seis meses após o TCTH (GUIDA M, et al., 2016).

\section{CONSIDERAÇÕES FINAIS}

Verificou-se que a DECH pode resultar em complicações ginecológicas severas para mulheres que se submeteram a TCTH. Uma das principais dificuldades avaliadas é a subnotificação dessas complicações e que, por isso, são pouco consideradas pelos ginecologistas. As pacientes que se submeteram a TCTH, portanto, devem realizar exames ginecológicos regularmente para manutenção de sua saúde. Considerando que os achados são majoritariamente clínicos e nem sempre evidenciados em biópsia, cabe aos ginecologistas se atentarem à conduta para com essas pacientes, orientando-as acerca de possíveis complicações, que muitas vezes são relevadas ante o desconhecimento da paciente. Sendo a paciente menina pré-púbere submetida a TCTH, é basilar que também seja realizado acompanhamento com endocrinologista. A adoção de condutas pré e pós-transplante é fundamental para evitar agravos.

\section{REFERÊNCIAS}

1. CIAVATtinI A, CLEMENTE N. Female Genital Tract Chronic Graft-Versus-Host Disease: Review of Literature. Anticancer Research, 2015; 35(1): 13-18.

2. CORSON SL, et al. Gynecologic manifestations of chronic graft-versus-host disease. Obstetrics \& Gynecology, 1982; 60(4): 488-92.

3. DYER G, et al. A survey of fertily and sexual health following allogenic haematopoietic stem cell transplantation in New South Wales, Australia. British Journal of Haematology, 2016; 172(4): 592-601.

4. FIGUEIREDO TB, et al. Reações adversas no dia zero do transplante de células-tronco hematopoéticas: revisão integrativa. Revista Gaúcha de Enfermagem, 2018; 39-95.

5. GUIDA M, et al. Reproductive issues in patients undergoing Hematopoietic Stem Cell Transplantation: an update. Journal of ovarian research, 2016; 9(1): 72.

6. HIRSCH P, et al. Female Genital Chronic Graft-Versus-Host Disease: Importance of Early Diagnosis to Avoid Severe Complications. Transplantation, 2012; 93: 1265-1269.

7. INSTITUTO NACIONAL DE CÂNCER (INCA). 2018. In: TRANSPLANTE de medula óssea. Brasil. Disponível em: https://www.inca.gov.br/tratamento/transplante-de-medula-ossea. Acesso em: 12 jan. 2021.

8. JACOBSON M, et al. Vulvovaginal graft-versus-host disease: a review. Climacteric, 2019; 22(6): 603-609.

9. JAGASIA MH, et al. National Institutes of Health Consensus Development Project on Criteria for Clinical Trials in Chronic Graft-versus-Host Disease: I. The 2014 Diagnosis and Staging Working Group report. Biology of blood and marrow transplantation: journal of the American Society for Blood and Marrow Transplantation, 2015; 21(3): 389-401.

10. KLASA L, et al. The management of gynecological complications in long-term survivors after allogeneic hematopoietic cell transplantation-a single-center real-life experience. Annals of Hematology. 2020; 99(6): 1361-1368.

11. KORNIK RI, RUSTAGI AS. Vulvovaginal Graft-Versus-Host Disease. Obstetrics and gynecology clinics of North America, 2017; 44(3): 475-492. 
12. LAMBERTINI M, et al. Cancer and fertility preservation: international recommendations from an expert meeting. BMC Medicine, 2016; 14(1).

13. LEV-SAGIE A, et al. Management and long-term consequences of genital graft versus host disease following hematopoietic stem cell transplantation. Bone Marrow Transplantation, 2020; 55(12): 2234-2243.

14. MACHADO AN, et al. Female genital tract chronic graft-versus-host disease: A narrative review. Hematology, Transfusion and Cell Therapy, 2019; 41(1): 69-75.

15. MAJHAIL NS, et al. Recommended screening and preventive practices for long-term survivors after hematopoietic cell transplantation. Bone Marrow Transplantation. 2012; 47(3): 337-41.

16. MURPHY J, et al. A Practical Guide to Gynecologic and Reproductive Health in Women Undergoing Hematopoietic Stem Cell Transplant. Biology of Blood and Marrow Transplantation, 2019; 25: 331-343.

17. NOERSKOV KH, et al. Sexual function 1-year after allogeneic hematopoietic stem cell transplantation. Bone Marrow Transplantation, 2016; 51(6): 833-840.

18. SMITH KE, et al. A prospective study of female genital chronic graft-versus-host disease symptoms, signs, diagnosis and treatment. Acta Obstetricia et Gynecologica Scandinavica, 2018; 97(9): 1122-1129.

19. SOARES AC. Manifestações orais da doença do enxerto contra o hospedeiro crônica: revisão sistemática de literatura Revista Brasileira de Odontologia, 2016; 73: 157-164.

20. STRATTON P et al. Vulvovaginal Chronic Graft-Versus-Host Disease With Allogeneic Hematopoietic Stem Cell Transplantation. Clinical e translational R pesquisa, 2012; 93: 1265-1269.

21. SUTANI A, et al. Gonadal failure among female patients after hematopoietic stem cell transplantation for nonmalignant diseases. Clinical Pediatric Endocrinology, 2019; 28(4): 105-112. 